\title{
Ecological and Evolutionary Stabilities of Biotrophism, Necrotrophism, and Saprotrophism
}

\author{
Sayaki U. Suzuki ${ }^{1, \star}$ and Akira Sasaki ${ }^{2}$ \\ 1. Division of Plant Disease Management, Central Region Agricultural Research Center, National Agriculture and Food Research \\ Organization, Kannondai, Tsukuba, Ibaraki 305-8666, Japan; 2. Department of Evolutionary Studies of Biosystems, SOKENDAI \\ (Graduate University for Advanced Studies), Hayama, Kanagawa 240-0193, Japan; and Evolution and Ecology Program, \\ International Institute for Applied Systems Analysis, A-2361 Laxenburg, Austria
}

Submitted April 24, 2018; Accepted February 12, 2019; Electronically published May 13, 2019

Online enhancements: appendixes.

\begin{abstract}
AвSTRACT: Fungi have multiple trophic behaviors, including biotrophism (parasitism on living hosts), necrotrophism (parasitism through killing host tissues), and saprotrophism (feeding on decaying organic matter). Historical classifications of plant pathogens are based on many different axes, including their trophic dependence on living and dead plants, their pathogenicity and mutualistic relationship to host plants, and their transmission pathways and infection mechanisms. Such diverse classifications sometimes conflict with each other. Clarifying the delineations among these groups would promote synthesis of fungal biology with current ecological and evolutionary concepts. To ask when biotrophic, necrotrophic, or saprotrophic fungi are maintained and favored by selection, we constructed an epidemiological model that describes the transitions between four states of host plants: susceptible living plant (S), infected living plant (I), uninfected dead plant (D), and infected dead plant, or plant residue (R). States $S$ and D represent two kinds of resource - living and dead plant tissues - for fungal inocula (I and R). We obtained values for the basic reproductive number $\left(R_{0}\right)$, which defines the persistence criteria of fungi. On the basis of our results, we propose four types of ecological groups, corresponding to the patterns of dependence on nutrient resources: (1) parasitismdependent fungi, characterized by their critical dependence on living plants; (2) saprotrophism-dependent fungi, characterized by their critical dependence on dead plants; (3) facultatively dependent fungi, which are neither parasitism nor saprotrophism dependent; and (4) doubly dependent fungi, which are neither wholly parasitism dependent nor wholly saprotrophism dependent. This grouping can be used to suggest principles for effective pest control. Our model also reveals simple conditions for the evolution of fungal trophic behaviors.
\end{abstract}

\footnotetext{
* Corresponding author; email: sayaki.u.suzuki@gmail.com.

ORCIDs: Suzuki, https://orcid.org/0000-0002-6744-8139; Sayaki, https:// orcid.org/0000-0003-3582-5865.

Am. Nat. 2019. Vol. 194, pp. 000-000. (C) 2019 by The University of Chicago. 0003-0147/2019/19401-58422\$15.00. All rights reserved. This work is licensed under a Creative Commons Attribution-NonCommercial 4.0 International License (CC BY-NC 4.0), which permits non-commercial reuse of the work with attribution. For commercial use, contact journalpermissions@press.uchicago.edu. DOI: $10.1086 / 703485$
}

We found that, in the absence of a trade-off between virulence and other life-history parameters, milder fungal virulence in living plants is always selected for if plant-fungus population dynamics are stable. However, with sufficiently strong necrotrophic transmission, the host population densities show sustained cycles, which promote the evolution of higher virulence. Epidemiological synthesis of diverse trophism in plant-fungi relationship in our model thus opens the way to discuss the evolution of fungal lifestyles as a function of ecological conditions.

Keywords: basic reproductive number, disease control, fungal ecology, plant pathogen, saprophyte, trophic niche.

\section{Introduction}

Plants are exploited by a wide variety of pathogens, including viruses, bacteria, fungi, and nematodes, but the vast majority are fungi (Agrios 2005). Fungi are heterotrophic organisms, and they have more diverse life cycles than other heterotrophs, such as animals. For example, a fungal species may have several alternative hosts and may have an anamorph (a mold-like asexual stage) and a teleomorph (mushroom-like sexual stage) in its life cycle (Anikster 1981; Wolfe 1984). Fungi may produce either spores or hyphae for growth, depending on the environmental conditions faced during their life cycles (Cooke and Rayner 1984; Agrios 2005). Many fungi possess not only the ability to parasitize living plants but also prominent saprotrophic ability, in which they take their nutrients from dead or decaying organic matter (Cooke and Rayner 1984; Agrios 2005; see table 1 for the definitions of key terms in mycology used in this article).

Researchers have long attempted to classify fungal behaviors (de Bary 1887; Thrower 1966; Brian 1967; Garrett 1970; Lewis 1973; Luttrell 1974; Parbery 1996). For example, classifying fungi by their methods of obtaining nutrients has yielded numerous groups (table 2), ranging from obligate biotroph to obligate saprotroph or obligate necrotroph. The first axis of classification is their nutritional de- 
Table 1: Glossary for key mycological terms used in the article

\begin{tabular}{ll}
\hline Term & \multicolumn{1}{c}{ Definition } \\
\hline Biotroph & Parasitic organism that survives only on living hosts \\
Necrotroph & Parasitic organism that kills host and feeds on the dead matter \\
Saprotroph & Organism that feeds on dead or decaying organic material \\
Endophyte & Microbe that lives inside host tissues at some stages of their lives without causing apparent harm to the host \\
\hline
\end{tabular}

pendency on living or dead plants: obligate biotrophs depend only on living plants for their nutrition, cannot live without living hosts (de Bary 1887; Lewis 1973), and cannot, in principle, be incubated on artificial media (Thrower
1966). In contrast, obligate saprotrophs depend entirely on dead or decaying organic matter for their nutrition and are, by definition, nonpathogenic to living plants (de Bary 1887; Lewis 1973). Obligate necrotrophs are placed

Table 2: Correspondence between our definitions and traditional categories suggested by Lewis (1973) and Luttrell (1974) and their redefinition by using our SIDR model

\begin{tabular}{|c|c|c|c|c|c|c|c|c|c|}
\hline \multirow[b]{2}{*}{ Traditional group } & \multicolumn{3}{|c|}{$\begin{array}{c}\text { Transmission } \\
\text { ability }^{\mathrm{a}}\end{array}$} & \multirow{2}{*}{$\begin{array}{c}\text { Persistence } \\
\text { condition }^{\mathrm{b}}\end{array}$} & \multicolumn{4}{|c|}{ Our classification $^{c}$} & \multirow[b]{2}{*}{ Examples } \\
\hline & $\beta_{\mathrm{IS}}$ & $\beta_{\mathrm{RS}}$ & $\beta_{\mathrm{RD}}$ & & 1 & 2 & 3 & 4 & \\
\hline $\begin{array}{l}\text { Obligate } \\
\quad \text { biotrophs }{ }^{\mathrm{d}, e, f}\end{array}$ & + & $0 /^{*}$ & 0 & $R_{0}^{\mathrm{II}}>1$ & + & - & - & - & $\begin{array}{l}\text { Puccinia graminis, Pseudoperonospora cubensis, } \\
\text { Uromyces pisi, Blumeria graminis, Uredinales spp. } \\
\text { (Wolfe 1984; Mathre 1997; Maier et al. 2003; } \\
\text { Duplessis et al. 2011; Kemen and Jones 2012); } \\
\text { mutualistic endophytes and mycorrhizal fungi } \\
\text { (Hyde and Soytong 2008) }\end{array}$ \\
\hline Hemibiotrophs ${ }^{\mathrm{e}}$ & + & + & 0 & $R_{0}^{\mathrm{II}}+R_{0}^{\mathrm{RI}}>1$ & + & - & - & - & $\begin{array}{l}\text { Guignardia bidwellii (Luttrell 1974; Parbery 1996); } \\
\text { Colletotrichum acutatum (Parbery 1996; Delaye et al. } \\
\text { 2013); Pyricularia/Magnaporthe oryzae (Webster and } \\
\text { Gunnell 1992; Kemen and Jones 2012) }\end{array}$ \\
\hline $\begin{array}{l}\text { Obligate } \\
\text { necrotrophs }\end{array}$ & 0 & + & 0 & $R_{0}^{\mathrm{RI}}>1$ & + & - & - & - & $\begin{array}{l}\text { Gaeumannomyces graminis var. graminis (Syn. } \\
\text { Ophiobolus graminis; Rao 1959; Lewis 1973) }\end{array}$ \\
\hline $\begin{array}{l}\text { Obligate } \\
\text { saprotrophs }^{\mathrm{d}}\end{array}$ & 0 & 0 & + & $R_{0}^{\mathrm{RR}}>1$ & - & + & - & - & $\begin{array}{l}\text { Aspergillus niger (Kabbage et al. 2015; De Silva et al. } \\
\text { 2016); Phanerochaete velutina, Hypholoma } \\
\text { fasciculare (Wells and Boddy 2002; Boddy et al. } \\
\text { 2009); nonpathogenic Fusarium (Kaur et al. 2010) }\end{array}$ \\
\hline $\begin{array}{l}\text { Facultative } \\
\quad \text { necrotrophs }{ }^{\mathrm{d}}\end{array}$ & 0 & + & + & $R_{0}^{\mathrm{RR}}+R_{0}^{\mathrm{RI}}>1$ & - & + & + & + & $\begin{array}{l}\text { Alternaria brassicicola, Alternaria alternata, } \\
\text { Fusarium solani, Fusarium oxysporum (Fan and } \\
\text { Köller 1998; Laluk and Mengiste 2010); } \\
\text { Verticillium dahliae (Rao 1959; Lewis 1973) }\end{array}$ \\
\hline $\begin{array}{l}\text { Facultative } \\
\text { saprotrophs } \\
\text { (facultative } \\
\text { biotrophs) })^{\mathrm{d}, \mathrm{g}}\end{array}$ & + & + & + & $R_{0}>1$ & + & + & + & + & $\begin{array}{l}\text { Facultative mycorrhizal fungi and facultative lichens } \\
\text { (Lewis 1973); Armillaria mellea, Armillaria ostoyae } \\
\text { (Mihail and Bruhn 2005) }\end{array}$ \\
\hline
\end{tabular}

a Transmission ability $\beta_{i j}$ from $i$ to $j$, where $i$ is either infected plants (I) or infective residue (R) and $j$ is either susceptible plants (S) or uninfected dead plants (D). In each entry $\beta_{i j}, 0$ indicates that $\beta_{i j}$ is 0 , a plus sign indicates that $\beta_{i j}$ is significantly large, and an asterisk indicates that transmission from $i$ to $j$ is possible but occurs only occasionally.

${ }^{\mathrm{b}}$ Conditions for persistence of each category, based on equation (2): $R_{0}>1$ with some $\beta_{i j} \approx 0$, as described in the third column. Throughout the table $\beta_{\mathrm{ID}}=0$ is assumed (and hence $R_{0}^{\mathrm{ID}}=1$; see eq. [3]).

${ }^{\mathrm{c}}$ New ecological categories based on our classification (see fig. 3 ). Type $1=$ parasitism-dependent fungi; type $2=$ saprotrophism-dependent fungi; type $3=$ facultatively dependent fungi; type $4=$ doubly dependent fungi. In each traditional group, a plus sign indicates that the type can occur, and a minus sign indicates that it cannot.

${ }^{d}$ Five traditional groups of fungi based on the work of Lewis (1973) and his verbal definition.

e A traditional group of fungi based on the work of Luttrell (1974) and his verbal definition. The "holobiotrophs" suggested by him are included in the obligate biotrophs.

${ }^{\mathrm{f}}$ The category of obligate biotrophs includes the type in which $\beta_{\mathrm{RS}}>0$. In this case, however, this transmission ability is restricted to infection from free-living inocula (resting spores), which cannot reproduce — and can survive only—on the dead plant body.

${ }^{\mathrm{g}}$ The persistence condition for facultative saprotrophs is $R_{0}$ (defined in eq. [2]) greater than 1 . 
at still another corner - they have an ability to infect living plants but depend entirely on dead tissues for their nutrition, which they kill after infection (de Bary 1887; Lewis 1973), and are therefore recognized as parasitoids or highly virulent pathogens. Here, “obligate” refers to a group's perfect dependence on either living plants or organic matter derived from dead plants as a nutrient resource. Classification of microbes that utilize both living and dead plants as nutrition is complicated. For example, saprophytes are further classified according to their pathogenicity to living plants: a saprophytic microbe that causes disease in living plants is said to be exhibiting facultative parasitism, whereas facultative saprotrophism is said to occur when microbes that usually behave as parasites are able to utilize nonliving materials (de Bary 1887; Brian 1967). Such a flexible strategy is thought to be an adaptation to unpredictable nutrient supply in the form of living and dead plant bodies and is ubiquitous among plant-infecting microbes. Complicated classification schemes and the proposed groups of plantinfecting microbes are listed in table 2. These historical classifications of plant pathogens are based on many different axes and not surprisingly sometimes conflict with each other. There is a need to clarify these groupings in a synthetic way from the perspective of current ecological and evolutionary concepts.

Although the physiologically contrasting traits of parasitism and saprotrophism by plant-infecting microbes have attracted attention, theoretical models that incorporate these two traits simultaneously have not yet been published. Here, we propose an epidemic model for plant-pathogenic microbes, taking into account both biotrophic and saprotrophic behavior, and try to reclassify various trophic strategies, including obligate parasitism (either biotrophic or necrotrophic) and obligate saprotrophism, as simply as possible from an epidemiological perspective, that is, on the basis of the conditions by which these pathogens invade microbe-free plant populations. We focus on how our dynamic classification can help in planning effective strategies for the eradication of plant-pathogenic microbes.

Another purpose of our study was to examine the evolution of the life-history parameters of plant-infecting microbes that affect their biotrophic, necrotrophic, and saprotrophic strategies. For example, the degree of pathogenicity against living plants (virulence) and the efficiency of utilizing dead tissue (saprobic efficiency) define the position of a fungus species on the parasitism-saprotrophism spectrum or, more precisely, on the biotrophism-necrotrophism-saprotrophism simplex. We theoretically examine the evolution of fungal traits, including the pathogenicity of microbes in infected plants, to identify the conditions under which a microbial species evolves into an obligate biotroph, an obligate saprotroph, an obligate necrotroph, or a form intermediate between them.

\section{SIDR: An Epidemiological Model for Bio-, Necro-, and Saprotrophic Infections}

Fungi in general can utilize living plants, dead plants, or both as resources. These two types of nutrient resources also give microbes habitats for growing and persisting. Fungi colonize the resources and form a complex with the infected resources throughout their infection cycles (Garrett 1951; Cooke and Rayner 1984). Here, we attempt to describe microbial reproduction on living or dead plants in epidemiological terms. Our model describes the transition of microbes among four epidemiological states of the host plants, namely, susceptible living plant (S), infected living plant (I), uninfected dead plant (D), and infective plant residue or infected dead plant (R). These states are classified by the presence (I and $\mathrm{R}$ ) or absence (S and $\mathrm{D}$ ) of microbes and the nutrient resources provided by either living plants ( $\mathrm{S}$ and $\mathrm{I}$ ) or dead plant material (D and R). We can thus also categorize these states by the two basic trophic types present, namely, a biotroph (made up of S and I) and a saprotroph (made up of D and R). States I and R act as sources of infection, whereas $S$ and $\mathrm{D}$ do not but instead are resources for potential new infections. We call this the dynamic the SIDR model, where the population densities of S, I, D, and R change with time as

$$
\begin{aligned}
& \frac{d S}{d t}=\left(r_{\mathrm{S}} S+r_{\mathrm{I}} I\right)\left(1-\frac{S+I}{K}\right)-\beta_{\mathrm{IS}} I S-\beta_{\mathrm{RS}} R S-\alpha_{\mathrm{S}} S, \\
& \frac{d I}{d t}=\beta_{\mathrm{IS}} I S+\beta_{\mathrm{RS}} R S-\alpha_{\mathrm{I}} I, \\
& \frac{d D}{d t}=\alpha_{\mathrm{S}} S-\beta_{\mathrm{ID}} I D-\beta_{\mathrm{RD}} R D-u_{\mathrm{D}} D \\
& \frac{d R}{d t}=\alpha_{\mathrm{I}} I+\beta_{\mathrm{ID}} I D+\beta_{\mathrm{RD}} R D-u_{\mathrm{R}} R .
\end{aligned}
$$

Figure 1 illustrates the transitions among states in the SIDR model. Susceptible and infected plants, $\mathrm{S}$ and I, reproduce, respectively, at rates $r_{\mathrm{S}}$ and $r_{\mathrm{I}}$. Newly produced plants are susceptible (i.e., there is no vertical transmission) and are subject to density-dependent reproduction; living plants have a carrying capacity of $K$. A susceptible plant $S$ dies at mortality rate of $\alpha_{\mathrm{S}}$ and becomes a dead plant without microbes (D), while an infected plant I dies at mortality rate of $\alpha_{\mathrm{I}}$ and becomes an infected dead plant with microbes (infectious residue, $\mathrm{R}$ ). Then, $\mathrm{D}$ and $\mathrm{R}$ are degraded at rates $u_{\mathrm{D}}$ and $u_{\mathrm{R}}$, respectively, to inorganic materials that microbes can no longer utilize. The rate $u_{\mathrm{R}}$ is interpreted as the rate at which infectious residue $\mathrm{R}$ is degraded. In the transmission process, this model has four infection rates via different routes of infection. The subscripts in the term $\beta_{i j}$ express the route of infection from $i$ to $j$. For example, $S$ can change to I by infection from I at a rate of $\beta_{\mathrm{IS}}$; that is, $\beta_{\mathrm{IS}}$ represents the transmission rate from $I$ to $S$ that contributes to a new in- 

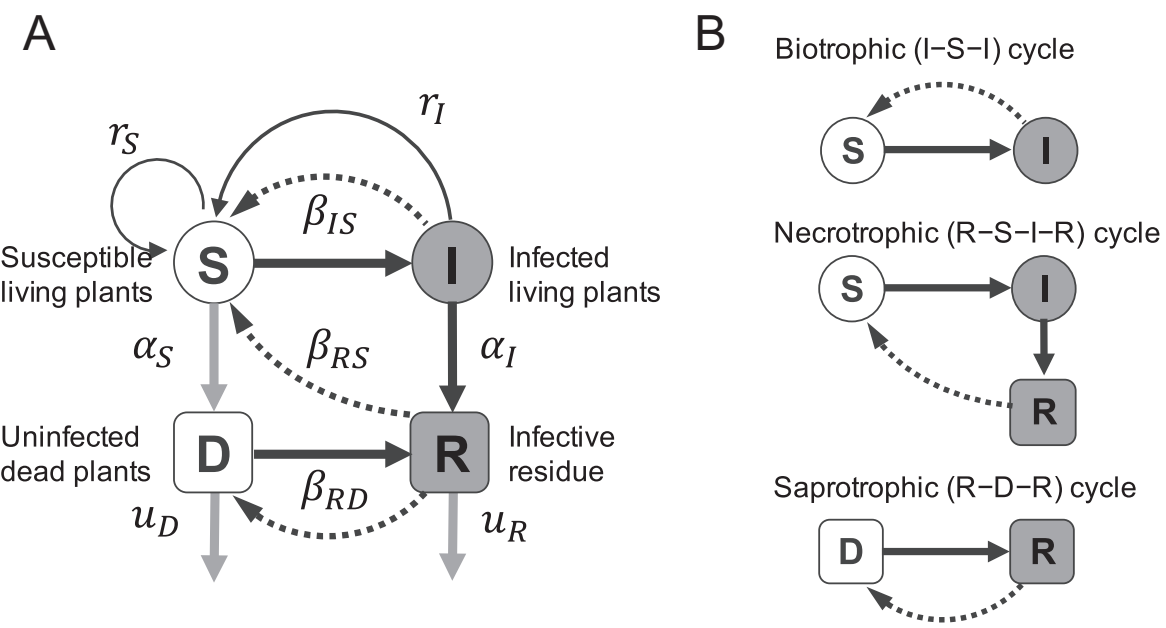

Figure 1: $A$, Schematic diagram of transitions of states in the SIDR model. Plant epidemic phases are categorized into four compartments: $\mathrm{S}$ (susceptible living plant), I (infected living plant), D (uninfected dead plant), and R (infected dead plant, or infective residue). There are four processes of transmission from I or R to S or D. See text for details. B, Three infection cycles in the SIDR model: biotrophic (I-S-I), necrotrophic (R-S-I-R), and saprotrophic (R-D-R).

fected living plant (I-S-I biotrophic cycle). Another route of infection exists as part of the same transition from $S$ to $I$ as a result of infection of a susceptible plant $S$ from infective residue (R) at the rate $\beta_{\mathrm{RS}}$-an infected living plant (I) thus produced will contribute to new infective residue $(\mathrm{R})$ to complete the necrotrophic cycle (R-S-I-R necrotrophic cycle). In the same fashion, an uninfected dead plant $\mathrm{D}$ changes to an infective dead plant $\mathrm{R}$ via two routes (I-D-R or R-D-R) at rates $\beta_{\mathrm{ID}}$ and $\beta_{\mathrm{RD}}$, respectively. Of these two, the saprotrophic (R-D-R) cycle is an important reproductive strategy in fungi, but the I-D-R pathway is difficult to distinguish from the death of an infected plant (though in some facultative saprotrophism, this infection pathway does exist), and it cannot complete an infection cycle by itself. Indeed, we show below that this I-D-R pathway does not affect either demographic or evolutionary dynamics, so our discussion on this pathway will be limited. This simple model can be used to describe various nutrient strategies of heterotrophic microbes depending on living and dead plants as resources.

\section{Results}

\section{Conditions for Invasion of Fungus-Free Host Populations by Fungi}

Here, we derive the conditions for invasion of a microbefree host population by infectious agents (I and R). The microbe-free equilibrium of equation (1) is defined as $(S, I, D, R)=\left(S_{0}, 0, D_{0}, 0\right)$, where

$$
\begin{aligned}
& S_{0}=K\left(1-\frac{\alpha_{\mathrm{s}}}{r_{\mathrm{s}}}\right) \text { and } \\
& D_{0}=\frac{\alpha_{\mathrm{S}}}{u_{\mathrm{D}}} K\left(1-\frac{\alpha_{\mathrm{s}}}{r_{\mathrm{S}}}\right)
\end{aligned}
$$

are the equilibrium densities of $\mathrm{S}$ and $\mathrm{D}$, respectively, with $I=R=0$. By constructing a next-generation matrix (Diekmann et al. 1990, 2010; Heesterbeek and Roberts 2007), we derive the overall basic reproductive number of fungi, $R_{0}$, which can be expressed in terms of individual $R_{0}^{i j}$ values, defined below in equation (4), as

$$
R_{0}=\frac{1}{2}\left[R_{0}^{\mathrm{II}}+R_{0}^{\mathrm{RR}}+\sqrt{\left(R_{0}^{\mathrm{II}}-R_{0}^{\mathrm{RR}}\right)^{2}+4 R_{0}^{\mathrm{IR}} R_{0}^{\mathrm{RI}}}\right]
$$

(see app. B for derivation; apps. A-D are available online). If $R_{0}<1$, the microbe-free equilibrium is stable, and if $R_{0}>1$, the equilibrium is unstable and microbes can invade the host population. Here, $R_{0}^{i j}$ represents four partial reproductive numbers of fungi, corresponding to four routes of microbial transmission from state $i$ to state $j$ :

$$
\begin{aligned}
R_{0}^{\text {bio }} \equiv R_{0}^{\mathrm{II}}=\frac{\beta_{\mathrm{IS}} S_{0}}{\alpha_{\mathrm{I}}} \quad(\mathrm{I}-\mathrm{S}-\mathrm{I} \text { cycle }), \\
R_{0}^{\text {sapro }} \equiv R_{0}^{\mathrm{RR}}=\frac{\beta_{\mathrm{RD}} D_{0}}{u_{\mathrm{R}}} \quad(\mathrm{R}-\mathrm{D}-\mathrm{R} \text { cycle }), \\
R_{0}^{\text {necro }} \equiv R_{0}^{\mathrm{RI}}=\frac{\beta_{\mathrm{RS}} S_{0}}{u_{\mathrm{R}}} \quad(\mathrm{R}-\mathrm{S}-\mathrm{I}-\mathrm{R} \text { cycle }), \quad \text { and } \\
R_{0}^{\mathrm{IR}}=\frac{\beta_{\mathrm{ID}} D_{0}}{\alpha_{\mathrm{I}}}+1 \quad(\mathrm{I}-\mathrm{D}-\mathrm{R} \text { pathway }),
\end{aligned}
$$

where $S_{0}$ and $D_{0}$ are the densities of susceptible living and dead plants, respectively, in the absence of microbes, defined above (eq. [2]).

As described in the parentheses in the equations, each $R_{0}^{i j}$ is identified by the combination of I and R in the super- 
script and represents a basic reproductive number, namely, the total number of secondary infected agents $j$ (in either I or $\mathrm{R}$ ) from a primary infected agent $i$ (again, either I or R) before it dies or is degraded (see fig. $1 B$ ). For example, $R_{0}^{\text {necro }} \equiv R_{0}^{\mathrm{RI}}$ is the expected number of living host plants secondarily infected from a single infective plant residue $\mathrm{R}$ before it is degraded into inorganic material; $R_{0}^{\text {bio }} \equiv R_{0}^{\mathrm{II}}$ and $R_{0}^{\text {sapro }} \equiv R_{0}^{\mathrm{RR}}$ are basic reproductive numbers defined only within the subpopulations of I and R, respectively, as seen in the feeding behaviors of some obligate biotrophs in the absence of dormant propagules on dead plants (having the I-S-I cycle only) and of obligate saprotrophs (having the R-D-R cycle only), respectively.

As stated above, a microbe cannot invade the population if its basic reproductive number is less than $1\left(R_{0}<1\right)$; this is equivalent, as shown in appendix $\mathrm{B}$, to the following inequalities (the shaded region in the $\left(R_{0}^{\text {bio }}+R_{0}^{\text {necro }}\right)-R_{0}^{\text {sapro }}$ plane in fig. 2):

$$
\begin{aligned}
\left(1-R_{0}^{\text {bio }}\right)\left(1-R_{0}^{\text {sapro }}\right) & >R_{0}^{\text {necro }} R_{0}^{\mathrm{IR}}, \\
\frac{\alpha_{\mathrm{I}}}{\alpha_{\mathrm{I}}+u_{\mathrm{R}}} R_{0}^{\text {bio }}+\frac{u_{\mathrm{R}}}{\alpha_{\mathrm{I}}+u_{\mathrm{R}}} R_{0}^{\text {sapro }} & <1 .
\end{aligned}
$$

Conversely, if inequality (5a) is violated, microbes can invade and can be maintained either in stable equilibrium or with limit cycles in the host population.

As shown in equation (4), either of the basic reproductive numbers for parasitic (biotrophic or necrotrophic) infection, $R_{0}^{\text {bio }}$ or $R_{0}^{\text {necro }}$, is proportional to the density of susceptible living plants, $S_{0}$, while that of saprotrophic infection, $R_{0}^{\text {sapro }}$, or that of I-D-R pathway, $R_{0}^{\mathrm{IR}}$, is proportional to the density of susceptible dead plants, $D_{0}$. Therefore, condition (5a) for the stability of microbe-free population defines the shaded region below a hyperbolic curve in the $S_{0}-D_{0}$ plane (fig. 2). Condition (5b) is necessary to exclude the alternative region outside the upper hyperbolic curve defined in condition (5a) from the microbe-free region (see eq. [A3]). We see that on

Biotrophic/necrotrophic basic reproductive number, $R_{0}^{\mathrm{II}}+R_{0}^{\mathrm{RI}}$

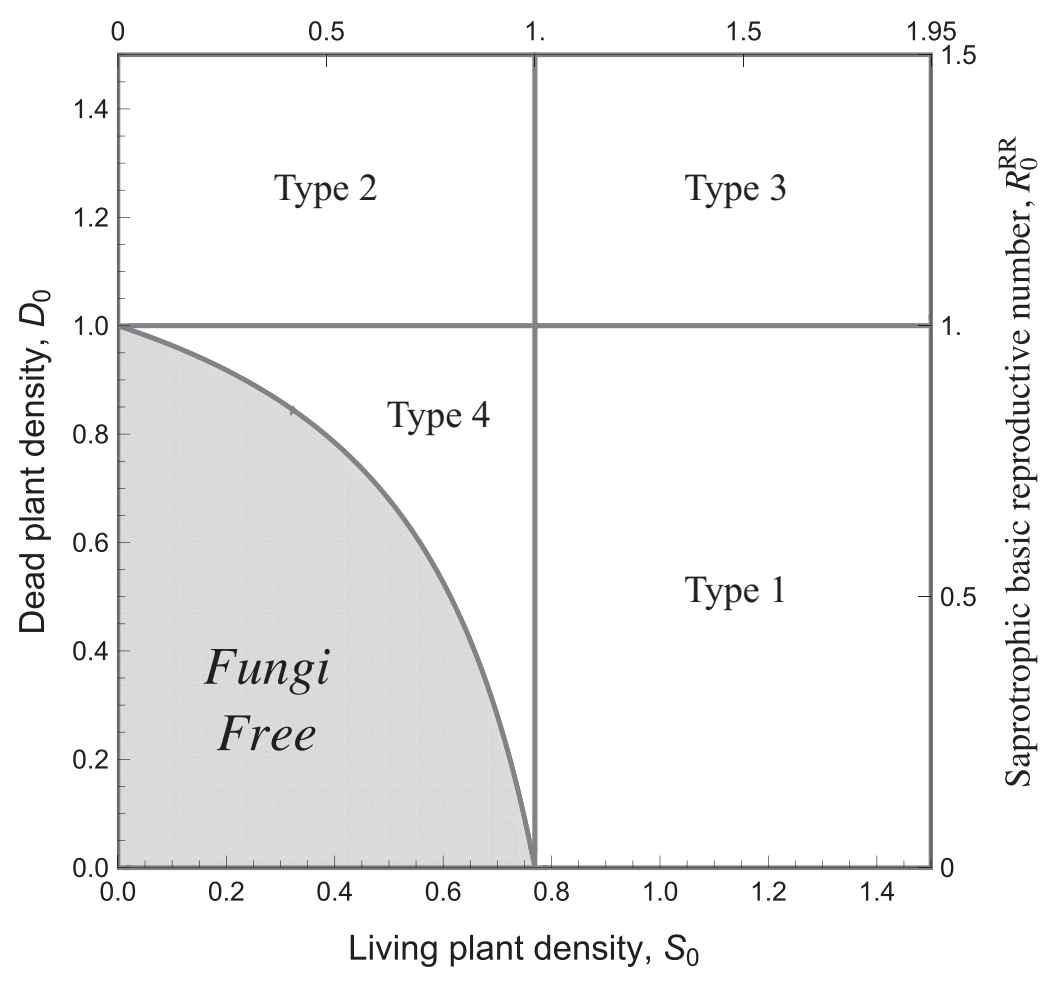

Figure 2: Classification of fungi according to their nutritional dependencies on living plants $S_{0}$ and dead plants $D_{0}$. Type 1 (parasitismdependent) fungi are those with biotrophic and necrotrophic basic reproductive numbers $R_{0}^{\text {bio }}+R_{0}^{\text {necro }} \equiv R_{0}^{\mathrm{II}}+R_{0}^{\mathrm{RI}}=\left(\beta_{\mathrm{IS}} / \alpha_{\mathrm{I}}+\beta_{\mathrm{RS}} / u_{\mathrm{R}}\right) S_{0}$ greater than 1 and a saprophytic basic reproductive number $R_{0}^{\text {sapro }} \equiv R_{0}^{\mathrm{RR}}=\left(\beta_{\mathrm{RD}} / u_{\mathrm{R}}\right) D_{0}$ less than 1 ; this means that they can be controlled by reducing the living-plant density alone. Type 2 (saprotrophism-dependent) fungi are those in the region $R_{0}^{\text {bio }}+R_{0}^{\text {necro }}<1$ and $R_{0}^{\text {sapro }}>1$ and can be controlled solely by removing dead plant bodies. Type 3 (facultatively dependent) fungi are those in the region $R_{0}^{\text {bio }}+R_{0}^{\text {necro }}>1$ and $R_{0}^{\text {sapro }}>1$; they can be controlled by reducing both living and dead plant body densities. Type 4 (doubly dependent) fungi are those in the region $R_{0}^{\text {bio }}+R_{0}^{\text {necro }}<1, R_{0}^{\text {sapro }}<1$, and $\left(S_{0}, D_{0}\right)$ that are outside the fungus-free area (shaded area). For all types, $\beta_{\mathrm{IS}} / \alpha_{\mathrm{I}}=1 ; \beta_{\mathrm{RS}} / u_{\mathrm{R}}=0.5$; $\beta_{\mathrm{ID}} / \alpha_{\mathrm{I}}=0.1 ;$ and $\beta_{\mathrm{RD}} / u_{\mathrm{R}}=1$. 
the $S_{0}$ axis where $D_{0}=0$, condition ( $5 \mathrm{a}$ ) reduces so that the basic reproductive number of parasitic infections is less than $1, R_{0}^{\text {bio }}+R_{0}^{\text {necro }}<1$, and that on the $D_{0}$ axis where $S_{0}=0$, it reduces so that the basic reproductive number of saprotrophic infection is less than $1, R_{0}^{\text {sapro }}<1$ (see alternative horizontal and vertical axes in fig. 2). Therefore, as discussed more fully in the next section, whether or not a microbe can be eradicated by reducing the densities of either susceptible living plants or susceptible dead plants depends on which of the four sections of figure 2 the microbe's parasitic $\left(R_{0}^{\text {bio }}+R_{0}^{\text {necro }}\right)$ and saprotrophic $\left(R_{0}^{\text {sapro }}\right)$ reproductive numbers are placed in.

\section{Trophic Dependencies of Microbes on Living and Dead Plants}

Fungi can successfully invade fungus-free populations and can be maintained stably if nutrient resources - either living or dead plants - are sufficiently abundant (the white region in the $\left(R_{0}^{\text {bio }}+R_{0}^{\text {necro }}\right)-R_{0}^{\text {sapro }}$ plane in fig. 2$)$. Here, we discuss the sensitivity of fungal persistence to parasitic (biotrophic or necrotrophic) and saprotrophic nutrition or to the population density $S_{0}$ of living plants and $D_{0}$ of dead plants. In the $\left(R_{0}^{\text {bio }}+R_{0}^{\text {necro }}\right)-R_{0}^{\text {sapro }}$ plane, where $R_{0}^{\text {bio }}+R_{0}^{\text {necro }}$ and $R_{0}^{\text {sapro }}$, respectively, indicate partial basic reproductive numbers through parasitic (biotrophic or necrotrophic) and saprotrophic nutrition, fungi can be classified into four groups, corresponding to the four regions in figure 2 (see also table 2).

Type 1 (Parasitism-Dependent) Fungi. These fungi can be controlled only by reducing living-plant density. (They are placed in the region of fig. 2 where the saprotrophic basic reproductive number is less than $1, R_{0}^{\text {sapro }}<1$, but the sum of biotrophic and necrotrophic basic reproductive numbers is greater than $1, R_{0}^{\text {bio }}+R_{0}^{\text {necro }}>1$.) By a decrease in the living-plant density $S_{0}, R_{0}^{\text {bio }}+R_{0}^{\text {necro }}$ can be moved to the region for extinction (gray area in fig. 2). Obligate biotrophs and hemibiotrophs (table 2), such as Uredinales (rusts), Blumeria graminis (powdery mildew), Peronosporaceae (downy mildews), and Pyricularia/Magnaporthe oryzae (rice blast), that cause crop diseases are typical examples of this group (Wolfe 1984; Webster and Gunnell 1992; Mathre 1997; Maier et al. 2003; Duplessis et al. 2011; Kemen and Jones 2012), as are mutualistic endophytes or mycorrhizal fungi (Hyde and Soytong 2008). This group also includes obligate necrotrophs, the basic reproductive number of which is represented as $R_{0}^{\text {necro }}$, such as Gaeumannomyces graminis (Rao 1959; Lewis 1973); see also table 2 for the relationship between our classification and the classical grouping of fungi.

Type 2 (Saprotrophism-Dependent) Fungi. These fungi can be controlled only by reducing dead-plant density. (They are placed in the region of fig. 2 where the sum of biotrophic and necrotrophic basic reproductive numbers is less than 1 , $R_{0}^{\text {bio }}+R_{0}^{\text {necro }}<1$, but the saprotrophic basic reproductive number is greater than $1, R_{0}^{\text {sapro }}>1$.) By a decrease in $D_{0}$ through removal of uninfected dead plants, $R_{0}^{\text {sapro }}$ can be moved to the region for extinction (gray area in fig. 2). Obligate saprotrophs, some facultative necrotrophs, and some facultative biotrophs belong to this group (table 2). Fungi with high necrotrophic growth ability, $R_{0}^{\text {necro }}>1$, do not belong to this group, as they can never be eradicated by reducing $D_{0}$ alone. Thus, the type 2 group consists of obligate saprotrophs (nonpathogenic) and opportunistic pathogens in facultative biotrophs and facultative necrotrophs. As a typical example, some members of the genus Armillaria have the ability to attack living trees, causing white rot (Mihail and Bruhn 2005). They depend on saprotrophic nutrition from decomposed wood; their parasitism (pathogenicity) is merely a measure aimed at breaking through the living cambial layer at all costs. Low-pathogenicity or nonpathogenic strains of facultative saprotrophs can be considered the main members of this group (Kaur et al. 2010).

Type 3 (Facultatively Dependent) Fungi. To control this group of fungi, one should reduce both living- and deadplant density. (In fig. 2, these fungi are placed in the region where $R_{0}^{\text {bio }}+R_{0}^{\text {necro }}>1$ and $R_{0}^{\text {sapro }}>1$.) Concomitant ability to exploit both living plants and dead plants could cause severe disease in agricultural fields. However, not many fungi simultaneously use both living and dead plants as resources during their life cycles (Luttrell 1974; Parbery 1996). For control, it therefore becomes important to target a growing stage that is specialized to either biotrophic or saprotrophic nutrition. Some facultative necrotrophs and facultative biotrophs belong to this category (table 2). A fungus belonging to this group may emerge if a highly virulent strain is selected from a facultative saprophyte under continuous cropping.

Type 4 (Doubly Dependent) Fungi. This group of fungi can be controlled by reducing either living- or dead-plant density. (In fig. 2, these fungi are placed in the region $R_{0}^{\text {bio }}+R_{0}^{\text {necro }}<1$ and $R_{0}^{\text {sapro }}<1$, but outside the area of extinction.) Some facultative necrotrophs and facultative biotrophs belong to this category (table 2). This group of fungi can maintain themselves only by the combined effect of biotrophic, necrotrophic, and saprotrophic transmission and not by any of these alone. Pathogenic fungi whose growth is limited by chemical control may fall conditionally in this category.

\section{Stable Endemic Equilibrium and Limit Cycles}

To discuss the stability of an endemic equilibrium and the evolution of epidemiological parameters, the following 
quantities, $\rho^{i j}$, defined for endemic equilibrium densities $\left(S^{*}, I^{*}, D^{*}, R^{*}\right)$ and corresponding to the basic reproductive numbers, $R_{0}^{i j}$, at microbe-free equilibrium $\left(S_{0}, 0, D_{0}, 0\right)$ defined in equation (3), are found to be important:

$$
\begin{aligned}
\rho^{\text {bio }} \equiv \rho^{\mathrm{II}} & =\frac{\beta_{\mathrm{IS}} S^{*}}{\alpha_{\mathrm{I}}}, \\
\rho^{\text {necro }} \equiv \rho^{\mathrm{RI}} & =\frac{\beta_{\mathrm{RS}} S^{*}}{u_{\mathrm{R}}}, \\
\rho^{\text {sapro }} \equiv \rho^{\mathrm{RR}} & =\frac{\beta_{\mathrm{RD}} D^{*}}{u_{\mathrm{R}}}, \quad \text { and } \\
\rho^{\mathrm{IR}} & =\frac{\beta_{\mathrm{ID}} D^{*}}{\alpha_{\mathrm{I}}}+1 .
\end{aligned}
$$

At an endemic equilibrium at which both plants and microbes exist with positive densities $\left(S^{*}, I^{*}, D^{*}, R^{*}\right)$, the following relationship, formally similar to condition (5) for the stability of disease-free (microbe-free) equilibrium, holds:

$$
\begin{gathered}
\frac{1}{2}\left(\rho^{\text {bio }}+\rho^{\text {sapro }}\right) \leq 1, \quad \text { and } \\
\left(1-\rho^{\text {bio }}\right)\left(1-\rho^{\text {sapro }}\right)=\rho^{\text {necro }} \rho^{\text {IR }} .
\end{gathered}
$$

If the condition (5) fails at the disease-free equilibrium, microbes can invade the population and be maintained either in a steady state or in a limit cycle. The steady state equilibrium tends to lose stability when necrotrophic reproduction is more efficient than biotrophic reproduction. For example, in the simplest case in which analytic calculation is tractable (where there is no infection from dead plants, $\beta_{\mathrm{ID}}=\beta_{\mathrm{RD}}=0$, and the infected host does not reproduce, $r_{\mathrm{I}}=0$ ), the endemic equilibrium becomes unstable and the population shows sustained cycles (i.e., a Hopf bifurcation occurs) if $\beta_{\mathrm{RS}}$ exceeds a threshold:

$$
\beta_{\mathrm{RS}}^{*}=\left(1-\frac{u_{\mathrm{R}}}{\alpha_{\mathrm{I}}}\right) \beta_{\mathrm{IS}}+\frac{r_{\mathrm{S}} \alpha_{\mathrm{I}}\left(r_{\mathrm{S}}-\alpha_{\mathrm{S}}+\alpha_{\mathrm{I}}\right)}{K\left(r_{\mathrm{S}}-\alpha_{\mathrm{S}}\right)\left(\alpha_{\mathrm{I}}-u_{\mathrm{R}}\right)}+O\left(\frac{1}{K^{2}}\right),
$$

where asymptotic expansion is for large $K$, with $O\left(1 / K^{2}\right)$ representing the terms of the order $1 / K^{2}$ or less for large $K$ (see app. C). The microbe population is then maintained in limit cycles, whereby bursts in the abundance of infected plants and infective residue lead to the depletion of susceptible plants and uninfected dead plants, followed by a decline in the abundance of infectious agents, before uninfected resources recover to a density sufficient to trigger the next burst of infectious agents. Phase diagrams of the demographic dynamics (eq. [1]) in a simplex of three transmission rates, namely, the biotrophic (I-S-I) transmission rate $\beta_{\mathrm{IS}}$, the necrotrophic (R-S-I-R) transmission rate $\beta_{\mathrm{RS}}$, and the saprotrophic (R-D-R) transmission rate $\beta_{\mathrm{RD}}$, under simple trade-offs between them are illustrated in figure 3, where $\beta_{\mathrm{ID}}=0$ is assumed. To simply illustrate how dynamical be- havior of the model depends on the biotrophic, necrotrophic, and saprotrophic transmission rates, we here assume that there is a trade-off, $\left[\left(\beta_{\mathrm{IS}}^{\sigma}+\beta_{\mathrm{RS}}^{\sigma}+\beta_{\mathrm{RD}}^{\sigma}\right) / 3\right]^{1 / \sigma}=$ constant (with $\sigma=0.5$ ), between biotrophic, necrotrophic, and saprotrophic transmission rates. It is also natural to assume that increasing one of the three transmission efficiencies will meet the decrease in the efficiencies of the others. With such a trade-off, the phase plane is divided into two regions (fig. 3) - when either the biotrophic or the saprotrophic transmission rate is large relative to the necrotrophic transmission rate, the microbe and plant populations are maintained in endemic equilibrium (top-right panel), whereas when the necrotrophic transmission rate is large relative to the biotrophic and saprotrophic transmission rates, the populations fluctuate cyclically in an asymptotic state (bottom-right panel). The boundary separating these two regions is the set of Hopf bifurcation points where the endemic equilibrium is destabilized. Similar phase diagrams are obtained for other values of $\sigma$ in the trade-off, including the case of linear trade-off, $\beta_{\mathrm{IS}}+\beta_{\mathrm{RS}}+\beta_{\mathrm{RD}}=$ constant (data not shown).

\section{Evolution of Virulence in Living Plants and Durability of Infectious Residue}

In our model, the difference between an endophyte and a necrotroph can be defined as the difference in severity of microbial virulence in the host, where virulence is defined as the additional death rate of infected plants $\alpha_{\mathrm{I}}-\alpha_{\mathrm{S}}$. We asked whether a mutant microbial genotype resulting in a different plant $\alpha_{\mathrm{I}}$ value could invade the endemic equilibrium population with the resident microbial genotype. The adaptive dynamics analysis in appendix A shows that a mutant causing an altered mortality rate, $\alpha_{\mathrm{I}}+\Delta \alpha_{\mathrm{I}}$, in infected plants could successfully invade and take over from an equilibrium population of microbial residents with a plant mortality rate of $\alpha_{\mathrm{I}}$ if and only if

$$
\left(\frac{\beta_{\mathrm{RS}} S^{*}}{u_{\mathrm{R}}}+\frac{\beta_{\mathrm{RD}} D^{*}}{u_{\mathrm{R}}}-1\right) \Delta \alpha_{\mathrm{I}}=\left(\rho^{\text {sapro }}+\rho^{\text {necro }}-1\right) \Delta \alpha_{\mathrm{I}}>0,
$$

where $S^{*}$ and $D^{*}$ are the densities of susceptible plants and uninfected dead plants, respectively, in endemic equilibrium with the resident fungal strain. If the mutant has lower virulence than the resident fungus $\left(\Delta \alpha_{\mathrm{I}}<0\right)$, this condition becomes $\beta_{\mathrm{RS}} S^{*} / u_{\mathrm{R}}+\beta_{\mathrm{RD}} D^{*} / u_{\mathrm{R}}<1$, or $\rho^{\text {sapro }}+\rho^{\text {necro }}<1$, where $\rho$ 's are the basic reproductive numbers at endemic equilibrium defined in equation (6). Thus, if the expected number of susceptible plants and dead plants secondarily infected from infective residue before it is degraded is less than 1 , then a mutant that behaves more mildly in the infected plants will be able to invade. Although equation (9) suggests that a more virulent fungus could invade if $\rho^{\text {sapro }}+\rho^{\text {necro }}>1$, 


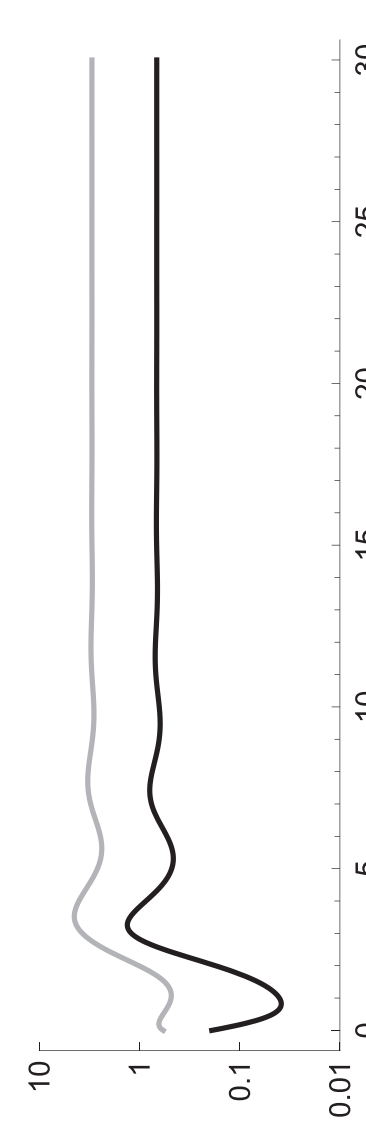

y pue I

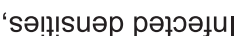

tে

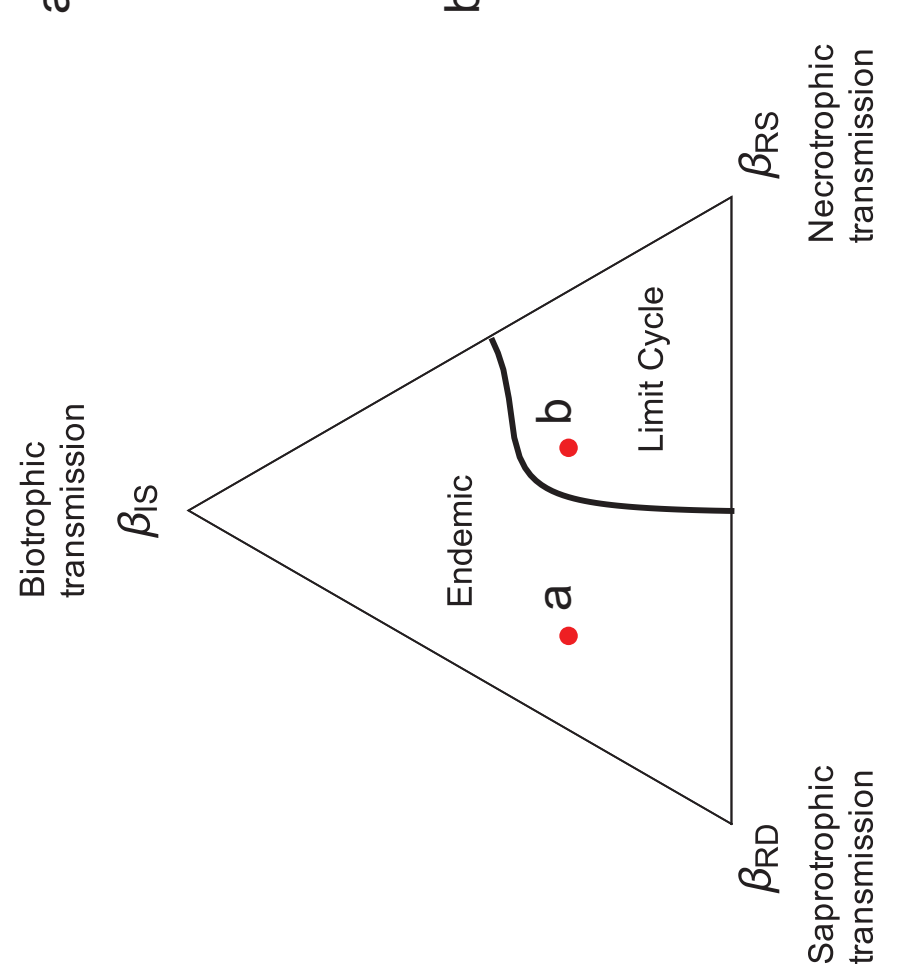

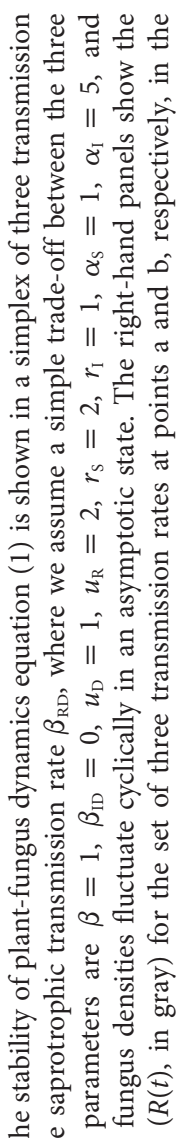

\& $2 己 \Xi$

声㝎芯艺

व

สี ิิ

ले

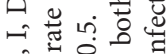

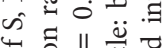

잉

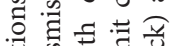

节节寻

\&

范芯

$\rightarrow$ के के के

bo

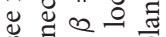

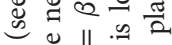

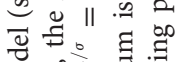

ले

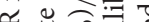

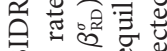

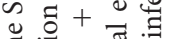

我获

पै

है

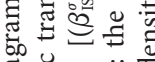

可

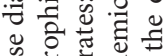

苋㻤

$\ddot{\sim} \approx$.

$\ddot{\sim} \Xi$ है

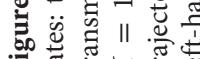

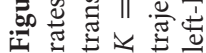

This content downloaded from 147.125.057.199 on May 28, 2019 05:18:11 AM 
we see below that this inequality is never satisfied in endemic equilibrium.

\section{Evolution toward Endophytism in Stable Endemic Populations}

From the invasiveness criteria (eq. [9]) of a mutant causing a mortality rate of $\alpha_{\mathrm{I}}+\Delta \alpha_{\mathrm{I}}$ in infected plants currently in endemic equilibrium with a resident fungus causing a plant mortality rate of $\alpha_{\mathrm{I}}$, we see that a mutant with low virulence $\left(\Delta \alpha_{\mathrm{I}}<0\right)$ can always invade the equilibrium resident fungal population, because $\rho^{\text {sapro }}+\rho^{\text {necro }}<1$ must be satisfied in endemic equilibrium. This is shown by noting that the finite growth rate of the population (the dominant eigenvalue $\rho^{*}$ of the next-generation matrix) must be equal to 1 at endemic equilibrium $\left(S^{*}, I^{*}, D^{*}, R^{*}\right)$ :

$$
\rho^{*}=\frac{1}{2}\left[\rho^{\text {bio }}+\rho^{\text {sapro }}+\sqrt{\left(\rho^{\text {bio }}-\rho^{\text {sapro }}\right)^{2}+4 \rho^{\text {necro }} \rho^{\mathrm{IR}}}\right]=1 .
$$

This implies that $\left(\rho^{\text {bio }}+\rho^{\text {sapro }}\right) / 2<1$ and $\left(1-\rho^{\text {bio }}\right)$ $\left(1-\rho^{\text {sapro }}\right)=\rho^{\text {necro }} \rho^{\text {IR }}$ hold in endemic equilibrium (app. A), which then implies that $\rho^{\text {sapro }}+\rho^{\text {necro }}<1$ and $\rho^{\text {bio }}<1$ (app. A). Therefore, $\rho^{\text {sapro }}+\rho^{\text {necro }}<1$ holds whenever the population stays in endemic equilibrium (i.e., when the endemic equilibrium is locally stable); hence, virulence always decreases evolutionarily (toward endophytism) as long as the endemic equilibrium is stable.

\section{Evolution toward Increased Durability of Infective Residue}

If the rate of degradation of infective residue $\left(u_{\mathrm{R}}\right)$ is subject to selection, a similar analysis leads to the condition under which a mutant with an inoculum degradation rate of $u_{\mathrm{R}}+$ $\Delta u_{\mathrm{R}}$ can invade an equilibrium population of resident fungi that have an inoculum degradation rate of $u_{\mathrm{R}}$ :

$$
\left(\frac{\beta_{\mathrm{IS}} S^{*}}{\alpha_{\mathrm{I}}}-1\right) \Delta u_{\mathrm{R}}=\left(\rho^{\text {bio }}-1\right) \Delta u_{\mathrm{R}}>0 .
$$

If the mutant has a degradation rate in infective residue slower than that of the resident infective residue $\left(\Delta u_{R}<0\right)$, this condition becomes $\beta_{\text {IS }} S^{*} / \alpha_{\mathrm{I}}<1$, or $\rho^{\text {bio }}<1$. Then, the fungus will evolve to decrease the rate at which infective residue is degraded or loses its infectivity. Although equation (11) suggests that a mutant with a higher $u_{\mathrm{R}}$ could invade if $\rho^{\text {bio }}>1$, this never happens in an endemic equilibrium, as shown in the previous section. Therefore, microbes always evolve toward increased durability of infective residue by reducing $u_{\mathrm{R}}$ in a stable endemic population.

These evolutionary analyses assume that the resident fungal population is in stable equilibrium. However, as equation (8) and figure 3 show, there is a parameter region in which the plant and fungus populations indefinitely fluctuate in density (i.e., they are in a limit cycle). We next examine numerically the evolution of virulence and the rate of degradation by fungi if the parameters of the resident fungus are in the limit cycle region.

\section{Evolution of Greater Virulence in Fluctuating Plant-Microbe Dynamics}

We thus found that the demographic stability of endemic equilibrium affects the evolution of fungus lifestyles. For evolution toward greater virulence (necrotrophs) or toward shorter durability of infective residue, it is necessary for the endemic equilibrium to be unstable. As we have shown in equation (7), endemic equilibrium loses its stability to yield limit cycles when the necrotrophic infection rate, $\beta_{\mathrm{RS}}$, is sufficiently large relative to the biotrophic in-

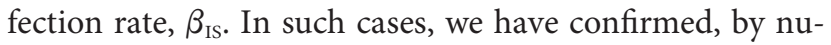
merical simulations of multistrain model with mutation between two adjacent genotypes differing slightly in virulence (see fig. 4 legend for details), that evolution toward greater virulence occurs even without a trade-off between virulence and transmission rate (fig. $4 A-4 C$ ). Figure $4 A$ shows an evolutionary trajectory in which the mean virulence of microbe population steadily increases when the densities of infected living plants and dead plants fluctuate. The underlying mechanism for this upward evolution of virulence is analyzed in figure $4 B, 4 C$, where the combined basic reproductive numbers of necrotrophic and saprotrophic cycles, $\rho^{\text {sapro }}(t)+\rho^{\text {necro }}(t)=\beta_{\mathrm{RD}} D(t) / u_{\mathrm{R}}+\beta_{\mathrm{RS}} S(t) / u_{\mathrm{R}}$, is plotted, which shows that the condition for the evolution of higher virulence, $\rho^{\text {sapro }}+\rho^{\text {necro }}>1$, is temporarily met in the shaded time splits (fig. $4 B$ ). During such time splits, the mean virulence indeed increases most steeply. Furthermore, we found that there is bistability in the evolution of virulence (fig. 4D). In other words, the mean virulence of a fungal population converges to either a high value or a low value, depending on the initial virulence of the population. The evolutionary trajectories converging to a high virulence are associated with limit cycles, and those converging to a low virulence are associated with stable equilibrium.

\section{Discussion}

We propose a simple model of the demographic dynamics of plants and fungi that can utilize both living and dead plant tissue. This model covers various lifestyles of plantdependent fungi, including biotrophs, necrotrophs, and saprotrophs, that can be classified according to the presence or absence of four routes of transmission between uninfected living plants and dead plants and infected plants and infective residues. Our model reveals the following. (1) The condition for the persistence of fungi can be expressed as the 

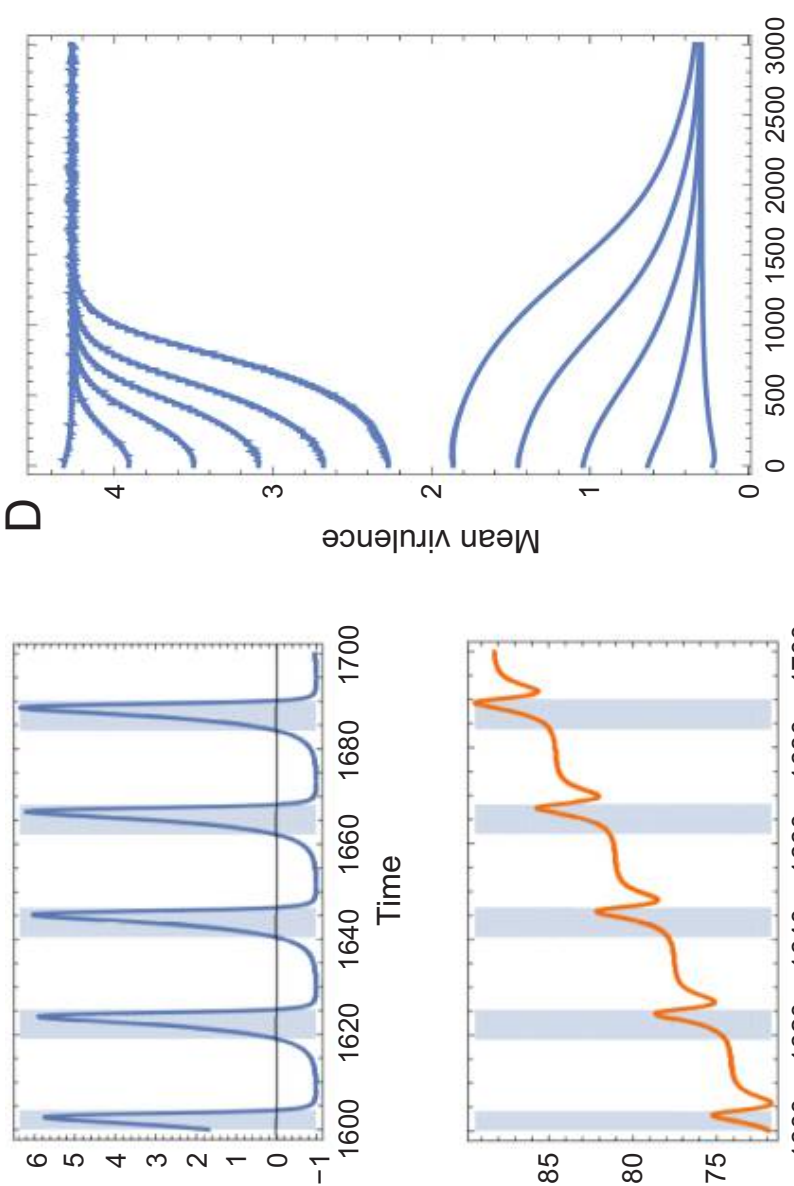

$1-(1)_{y y} d+(\mathfrak{l})_{1 y} d$

m

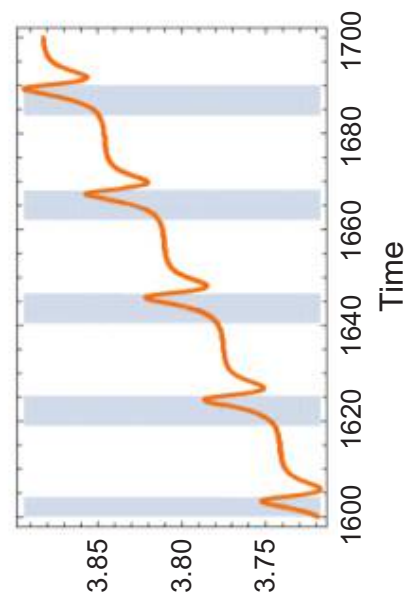

әэиə|กม!^ иеәW

u

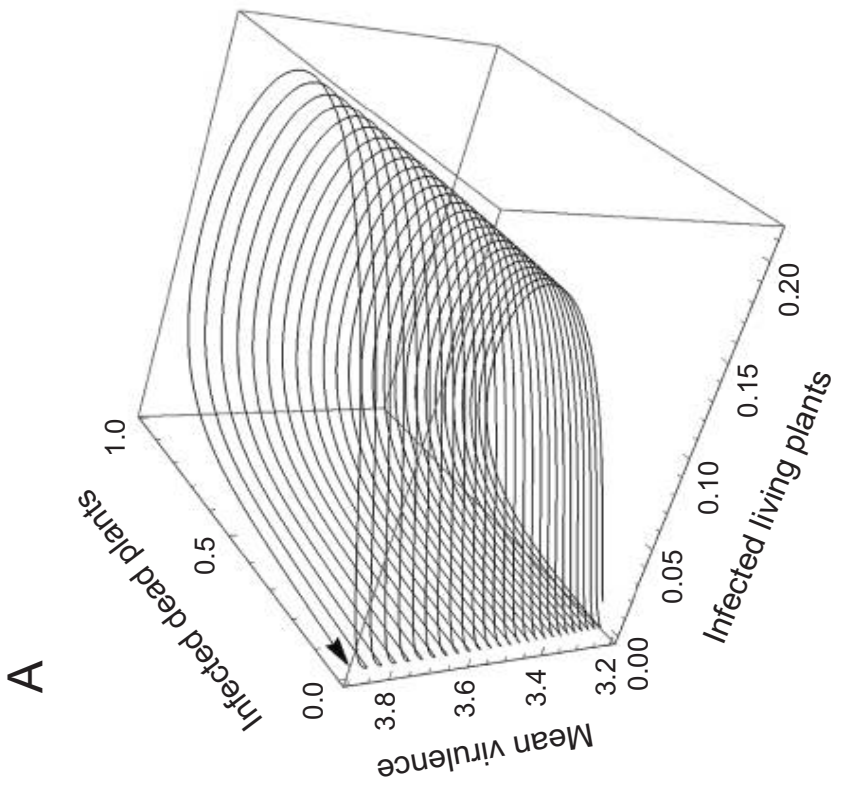

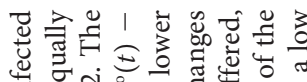

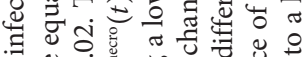

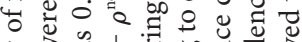

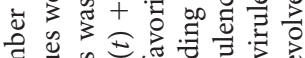

(1)

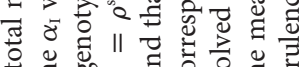

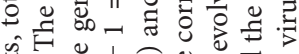

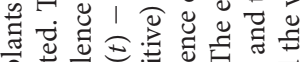

on

I. $5+2.5$

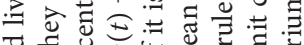

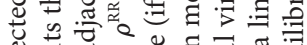

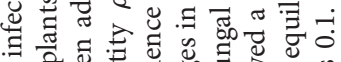

पू.

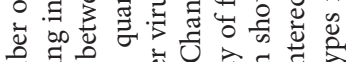

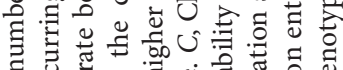

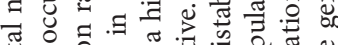

\% ४ु

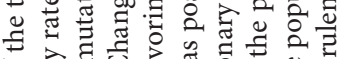

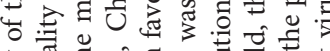

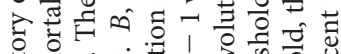

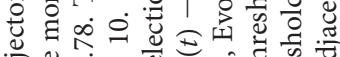

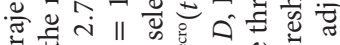

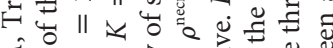

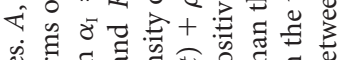

过司

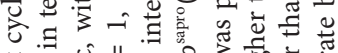

青它

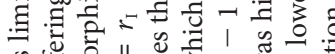

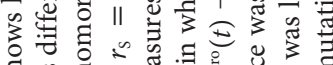

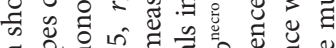

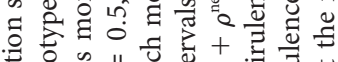

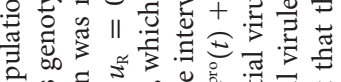

客品.

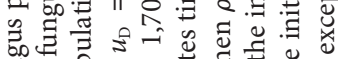

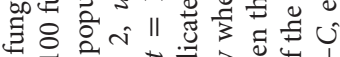

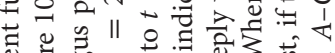

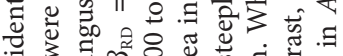

क्ष

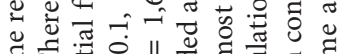

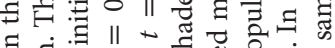

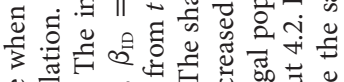

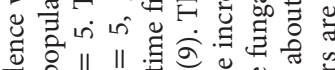

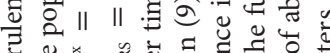

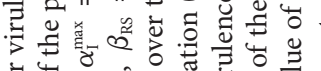

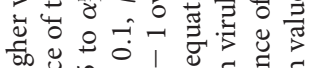

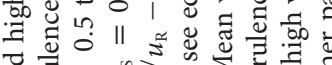

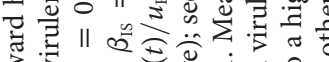

ริ 500 \%

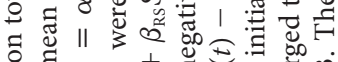

है

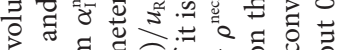

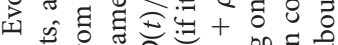

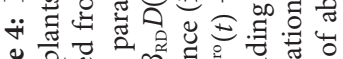

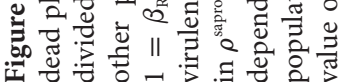

This content downloaded from 147.125.057.199 on May 28, 2019 05:18:11 AM 
combination of four basic reproductive numbers that correspond to four transmission routes of fungi between living and dead plants. (2) The combined values of these numbers can tell us whether we can eradicate fungi by removing living plants or dead plants; hence, they offer a theoretical basis for the effective control of fungal infections in natural and crop plants. These numbers also redefine the traditional classifications of fungi. (3) In endemic equilibrium, the corresponding four basic reproductive numbers determine whether a mutant strain causing greater or less mortality in infected plants (i.e., with high or low virulence) can invade and displace the resident fungal population. More specifically, if the sum of two basic reproductive numbers $\left(\rho^{\text {bio }}+\rho^{\text {necro }}\right)$, that is, the sum of those through the necrotrophic cycle and through the saprotrophic cycle from infective residue, is less than 1 (i.e., if the total basic reproductive ratio of fungi in dead infected plants is less than 1), less virulence to living plants will evolve in fungi. Conversely, if this value is greater than 1, greater virulence will evolve. (4) A similar relationship exists between conditions for the evolution of longer durability of infective residue and basic reproductive numbers in saprotrophic or biotrophic cycles: if $\rho^{\text {bio }}>1$ (i.e., $\rho^{\text {sapro }}<1$ ), shorter durability of infective residue evolves, whereas if the reverse inequality holds, longer durability evolves. (5) The demographic stability of the endemic equilibrium of the resident fungal population affects the invasiveness of a mutant fungal strain: only a mutant with less mortality in infected plants and greater durability of infective residue can invade if the endemic equilibrium is stable in the resident population. However, if the endemic equilibrium is unstable and the fungal and plant population densities fluctuate (i.e., show limit cycles), then a mutant with greater virulence and shorter durability of infective residue can invade.

It is well known that if there is no trade-off between virulence (the excess mortality of infected hosts) and other epidemiological parameters such as transmission or recovery rate, then virulence evolves to its minimum in the epidemic models of infectious diseases in animal and humans (Anderson and May 1991). This is because higher virulence simply reduces the mean infectious period of an infected host and hence reduces the basic reproductive number. In our model of plant-pathogen interaction, with biotrophic, necrotrophic, and saprotrophic infection pathways, higher virulence, or higher mortality of an infected living plant, leads to a shorter infectious period in the living plants but also to earlier production of infectious dead plants, which may improve the efficiency of necrotrophic or saprotrophic infection cycles. Therefore, even without any trade-off between virulence and transmission rates (or other parameters), the evolution of virulence becomes an interesting subject to study theoretically. Our finding, however, is rather simple if the population is in endemic equilibria: milder viru- lence in living plants is always selected for in plant-infecting microbes, irrespective of the presence of a necrotrophic or saprotrophic pathway. However, once the endemic population is destabilized, as a result of a sufficient effect of the microbe on necrotrophic transmissions, higher virulence could be selected for even without any trade-off. Now, earlier production of infectious dead plants by higher virulence does improve the efficiency of necrotrophic infection cycles, amplifying the amplitude of the sustained cycles and leading to evolution toward even higher virulence (necrotrophic spiral; fig. 4). This self-reinforcement toward necrotrophism is responsible for our finding of evolutionary bistability in virulence: the virulence increases if the initial virulence is high enough to ensure that the population cycles, while it evolves to a minimum if the initial virulence is lower than a threshold to destabilize the endemic equilibrium.

In some highly virulent plant-infecting fungi, such as ergot fungi and false smut of rice (Lewis 1973; Luttrell 1974), killing hosts is closely associated with their spore dispersal, and hence they are regarded as obligate killer parasites that first grow within an infected host and then kill the host to release transmissible propagules (Ebert and Weisser 1997). Such parasites do not rely on dead material as a nutrient resource and hence are biotrophs. However, as they release most of infectious propagules after they kill the host, the majority of them are transmitted necrotrophically. The phase changes of pathogen behavior after infection complicate their classification and our understanding of their control and evolution. To understand the full spectrum of evolutionary trends in plant pathogens, including such ambiguous fungi, more evolutionary analyses should be developed in models that take into account within-host pathogen growth schedule (e.g., Sasaki and Iwasa 1991) and propagule release timing (Ebert and Weisser 1997).

Instability of endemic equilibrium occurs in our model when necrotrophic transmission is sufficiently intense relative to biotrophic or saprotrophic transmission. This is due to the presence of an intermediate compartment, infected living plants, between susceptible living plants and infectious dead plants in the necrotrophic infection cycle. This can be compared with both the instability that occurs in the epidemic dynamics with free-living infectious propagules (Anderson and May 1991) and the models of immune priming against pathogens of invertebrate hosts (Best et al. 2012; Tidbury et al. 2012). In the latter, the presence of immune-primed hosts induced by a certain proportion of contacts between susceptible and infected hosts destabilizes the epidemiological dynamics. As in our model where demographic stability affected the evolution of virulence, Best et al. (2012) found that, when the host trait of immune-priming efficiency is subject to selection, the evolutionarily stable proportion of primed hosts upon contact with an infected host and its evolutionary branching pat- 
terns are affected by sustained cycles induced by immune priming.

Researchers have performed phylogenetic analyses of the evolution of fungal lifestyles in endophytes, biotrophs, and necrotrophs (Andrew et al. 2012; Delaye et al. 2013). Endophytes are those groups of fungi (e.g., Colletotrichum gloeosporioides), actinomycetes, or bacteria that live inside host tissues at some stages of their lives without causing apparent harm to the host (Petrini 1991; Wilson 1995; Hyde and Soytong 2008) and act as mutualists or commensals. Delaye et al. (2013) showed several evolutionary changes from an endophytic lifestyle to a necrotrophic lifestyle - and changes in the opposite direction - using a maximum likelihood analysis combined with ancestral character mapping by parsimony. By contrast, biotrophs formed several independent clusters that did not contain endophytes or necrotrophs. Delaye et al. (2013, p. 125) concluded that "biotrophy usually represents a derived and evolutionarily stable trait, whereas fungi easily can switch between an endophytic and necrotrophic lifestyle at the evolutionary and even the ecological timescale." Our evolutionary theoretical analysis reveals that demographic stability in plant-pathogen epidemic dynamics affects whether the pathogen evolves toward a necrotrophic lifestyle having a high virulence in living plants or toward endophytism, which is harmless to living plants. As the stability of plant-microbe endemic equilibrium depends on the transmission efficiencies of biotrophic, necrotrophic, and saprotrophic infection pathways, which should be affected by environmental conditions, the switch between necrotroph and endophyte could be triggered by environmental changes. Interestingly, there are many reported cases of the shifts from endophyte to necrotrophic pathogen caused, for example, by changes in environmental conditions (Hyde and Soytong 2008; Porras-Alfaro and Bayman 2011; Delaye et al. 2013).

Although we cannot explain all the evolutionary trends revealed in fungal lifestyles by phylogenetic analyses (Delaye et al. 2013), our model shows a role for demographic stability in directional evolution toward less virulent fungi (biotrophs or endophytes) and more virulent fungi (necrotrophic pathogens). Full evolutionary analyses, including an examination of transmission rates and trade-offs between them, were not examined in detail here, but our results suggest that the partial basic reproductive numbers provided here for each infection pathway are the key to understanding the evolutionary shifts between diverse fungal lifestyles.

Another contribution of our study is the redefinition of fungal groups in terms of their nutritional dependencies on living and dead plants. Although this axis for grouping fungi is basically shared with those of Lewis (1973) and Luttrell (1974), our classification based on demographic models is more efficient for grouping the ecological functions of fungi and clarifying the conditions required for their eradication or conservation. Table 2 and figure 5 summarize the conven-

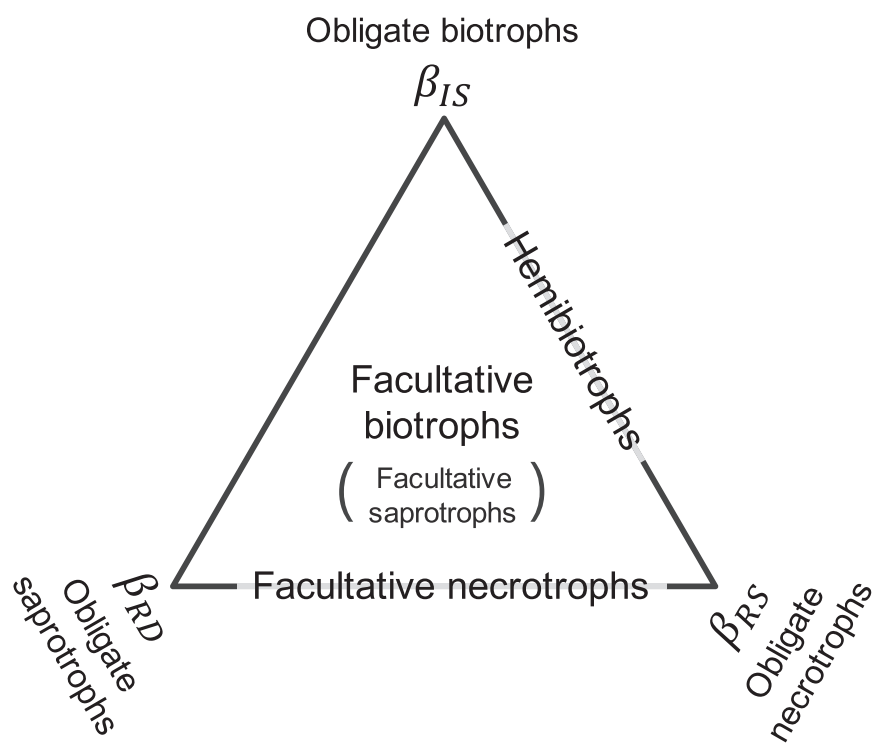

Figure 5: Schematic diagram of conventional fungal trophic grouping based on three transmission modes. This diagram represents the relative strength of three transmission parameters of fungi in a 3-simplex: $\beta_{\mathrm{IS}}$ (biotrophic transmission), $\beta_{\mathrm{RD}}$ (saprotrophic transmission), and $\beta_{\mathrm{RS}}$ (necrotrophic transmission). The conventional grouping by Lewis (1973) and Luttrell (1974) can be mapped onto the triangular parameter space (see table 2). Since obligate bio-/nacro-/saprotrophs have only one trophic mode, they are placed at the vertices of the triangle. Hemibiotrophs reside on the edge connecting vertex $\beta_{\mathrm{IS}}$ and vertex $\beta_{\mathrm{RD}}$. Likewise, facultative necrotrophs reside on the edge connecting vertex $\beta_{\mathrm{RS}}$ and vertex $\beta_{\mathrm{RD}}$. Since facultative biotrophs have every trophic mode, they are located inside the triangle. 
tional grouping of fungi by Lewis (1973) and Luttrell (1974), compared with our model's new interpretations using epidemic parameters. Lewis proposed five fungal groups based on nutritional and ecological behaviors: obligate saprotrophs, obligate necrotrophs, obligate biotrophs, facultative necrotrophs, and facultative biotrophs. Lewis's five groups are then redefined according to their dependence on three infection cycles (biotrophic, necrotrophic, and saprotrophic). This reinterpretation allows us to have the explicit persistence condition and the eradication criterion in terms of the three basic reproductive numbers $\left(R_{0}^{\text {bio }}, R_{0}^{\text {necro }}\right.$, and $\left.R_{0}^{\text {sapro }}\right)$, and their expected evolutionary trends, as summarized in appendix D.

In this article, we tried to classify previously defined groups of fungi ranging between obligate biotroph, obligate necrotroph, and obligate saprophyte by combinations of the presence or absence of four types of transmission ability. Epidemiological analysis of plant-microbe relationship in our model reveals the conditions for ecological persistence and eradication and opens the way to discuss the evolution of fungal lifestyles as a function of ecological conditions. Although our analysis revealed several conditions under which evolution toward endophytes, necrotrophs, and saprotrophs is favored, they only scratch the surface of comprehensive theoretical understanding of the evolution of diverse fungal lifestyles requires future studies.

\section{Acknowledgments}

We thank Yoh Iwasa and Hiroshi Nishiura for their helpful comments. This work was supported in part by a grant-in-aid to A.S. from MEXT (Ministry of Education, Culture, Sports, Science and Technology, Japan).

\section{Literature Cited}

Agrios, G. N. 2005. Plant pathology. 5th ed. Academic Press, San Diego, CA.

Anderson, R. M., and R. M. May. 1991. Infectious diseases of humans: dynamics and control. Oxford University Press, Oxford.

Andrew, M., R. Barua, S. M. Short, and L. M. Kohn. 2012. Evidence for a common toolbox based on necrotrophy in a fungal lineage spanning necrotrophs, biotrophs, endophytes, host generalists and specialists. PLoS One 7:e29943. doi:10.1371/journal.pone.0029943.

Anikster, Y. 1981. Alternate hosts of Puccinia hordei. Phytopathology 72:733-735.

Best, A., H. Tidbury, A. White, and M. Boots. 2012. The evolutionary dynamics of within-generation immune priming in invertebrate hosts. Journal of the Roval Society Interface 10:20120887. doi:10 .1098/rsif.2012.0887.

Boddy, L., J. Hynes, D. P. Bebber, and M. D. Fricker. 2009. Saprotrophic cord systems: dispersal mechanisms in space and time. Mycoscience 50:9-19.

Brian, P. W. 1967. The Leeuwenhoek Lecture, 1966: obligate parasitism in fungi. Proceedings of the Roval Societv B 168:101-118.
Cooke, R. C., and A. D. M. Rayner. 1984. Ecology of saprotrophic fungi. Longman, London.

de Bary, A. 1887. Comparative morphology and biology of the fungi, mycetozoa and bacteria. H. E. F. Garnsey and I. B. Balfour, trans. Clarendon, Oxford.

Delaye, L., G. García-Guzmán, and M. Heil. 2013. Endophytes versus biotrophic and necrotrophic pathogens - are fungal lifestyles evolutionarily stable traits? Fungal Diversity 60:125-135.

De Silva, N. I., S. Lumyong, K. D. Hyde, T. Bulgakov, A. J. L. Phillips, and J. Y. Yan. 2016. Mycosphere essays 9: defining biotrophs and hemibiotrophs. Mycosphere 7:545-559.

Diekmann, O., J. A. P. Heesterbeek, and J. A. J. Metz. 1990. On the definition and the computation of the basic reproduction ratio $R_{0}$ in models for infectious diseases in heterogeneous populations. Journal of Mathematical Biology 28:365-382.

Diekmann, O., J. A. P. Heesterbeek, and M. G. Roberts. 2010. The construction of next-generation matrices for compartmental epidemic models. Journal of the Roval Societv Interface 7:873-885.

Duplessis, S., C. A. Cuomo, Y.-C. Lin, A. Aerts, E. Tisserant, C. Veneault-Fourrey, D. L. Joly, et al. 2011. Obligate biotrophy features unraveled by the genomic analysis of rust fungi. Proceedings of the National Academy of Sciences of USA 108:9166-9171.

Ebert, D., and W. W. Weisser. 1997. Optimal killing for obligate killers: the evolution of life histories and virulence of semelparous parasites. Proceedings of the Roval Society B 264:985-991.

Fan, C.- Y., and W. Köller. 1998. Diversity of cutinases from plant pathogenic fungi: differential and sequential expression of cutinolytic esterases by Alternaria brassicicola. FEMS Microbiology Letters 158:33-38.

Garrett, S. D. 1951. Ecological groups of soil fungi: a survey of substrate relationships. New Phvtologist 50:149-166.

1970. Pathogenic root-infecting fungi. Cambridge University Press, Cambridge.

Heesterbeek, J. A. P., and M. G. Roberts. 2007. The type-reproduction number $T$ in models for infectious disease control. Mathematical Biosciences 206:3-10.

Hyde, K. D., and K. Soytong. 2008. The fungal endophyte dilemma. Fungal Diversity 33:163-173.

Kabbage, M., O. Yarden, and M. B. Dickman. 2015. Pathogenic attributes of Sclerotinia sclerotiorum: switching from a biotrophic to necrotrophic lifestyle. Plant Science 233:53-60.

Kaur, A., J. Kaur, and R. S. Singh. 2010. Nonpathogenic Fusarium as a biological control agent. Plant Pathology Journal 9:79-91.

Kemen, E., and J. D. G. Jones. 2012. Obligate biotroph parasitism: can we link genomes to lifestyles? Trends in Plant Science 17:448457.

Laluk, K., and T. Mengiste. 2010. Necrotroph attacks on plants: wanton destruction or covert extortion? The Arabidopsis Book 8:e0136. doi:10.1199/tab.0136.

Lewis, D. H. 1973. Concepts in fungal nutrition and the origin of biotrophy. Biological Reviews 48:261-278.

Luttrell, E. S. 1974. Parsitism of fungi on vascular plants. Mycologia 66:1-15.

Maier, W., D. Begerow, M. Weiß, and F. Oberwinkler. 2003. Phylogeny of the rust fungi: an approach using nuclear large subunit ribosomal DNA sequences. Canadian Journal of Botany 81:12-23.

Mathre, D. E. 1997. Compendium of barley diseases. 2nd ed. American Phytopathological Society, St. Paul, MN.

Mihail, J. D., and J. N. Bruhn. 2005. Foraging behaviour of Armillaria rhizomorph systems. Mycological Research 109:1195-1207. 
Parbery, D. G. 1996. Trophism and the ecology of fungi associated with plants. Biological Reviews 71:473-527.

Petrini, O. 1991. Fungal endophytes of tree leaves. Pages 179-197 in J. H. Andrews and S. S. Hirano, eds. Microbial ecology of leaves. Springer, New York.

Porras-Alfaro, A., and P. Bayman. 2011. Hidden fungi, emergent properties: endophytes and microbiomes. Annual Review of Phvtopathology 49:291-315.

Rao, A. S. 1959. A comparative study of competitive saprophytic ability in twelve root-infecting fungi by an agar plate method. Transactions of the British Mycological Society 42:97-111.

Sasaki, A., and Y. Iwasa. 1991. Optimal growth schedule of pathogens within a host: switching between lytic and latent cycles. Theoretical Population Biology 39:201-239.

Thrower, L. B. 1966. Terminology for plant parasites. Journal of Phytopathology 56:258-259.

Tidbury, H. J., A. Best, and M. Boots. 2012. The epidemiological consequences of immune priming. Proceedings of the Roval Societv B 279:4505-4512.
Webster, R. K., and P. S. Gunnell. 1992. Compendium of rice diseases. American Phytopathological Society, St. Paul, MN.

Wells, J. M., and L. Boddy. 2002. Interspecific carbon exchange and cost of interactions between basidiomycete mycelia in soil and wood. Functional Ecology 16:153-161.

Wilson, D. 1995. Endophyte: the evolution of a term, and clarification of its use and definition. Oikos 73:274-276.

Wolfe, M. S. 1984. Trying to understand and control powdery mildew. Plant Pathology 33:451-466.

\section{References Cited Only in the Online Enhancements}

Gan, P., K. Ikeda, H. Irieda, M. Narusaka, R. J. O’Connell, Y. Narusaka, Y. Takano, Y. Kubo, and K. Shirasu. 2013. Comparative genomic and transcriptomic analyses reveal the hemibiotrophic stage shift of Colletotrichum fungi. New Phvtologist 197:1236-1249.

Associate Editor: Tom E. X. Miller Editor: Alice A. Winn

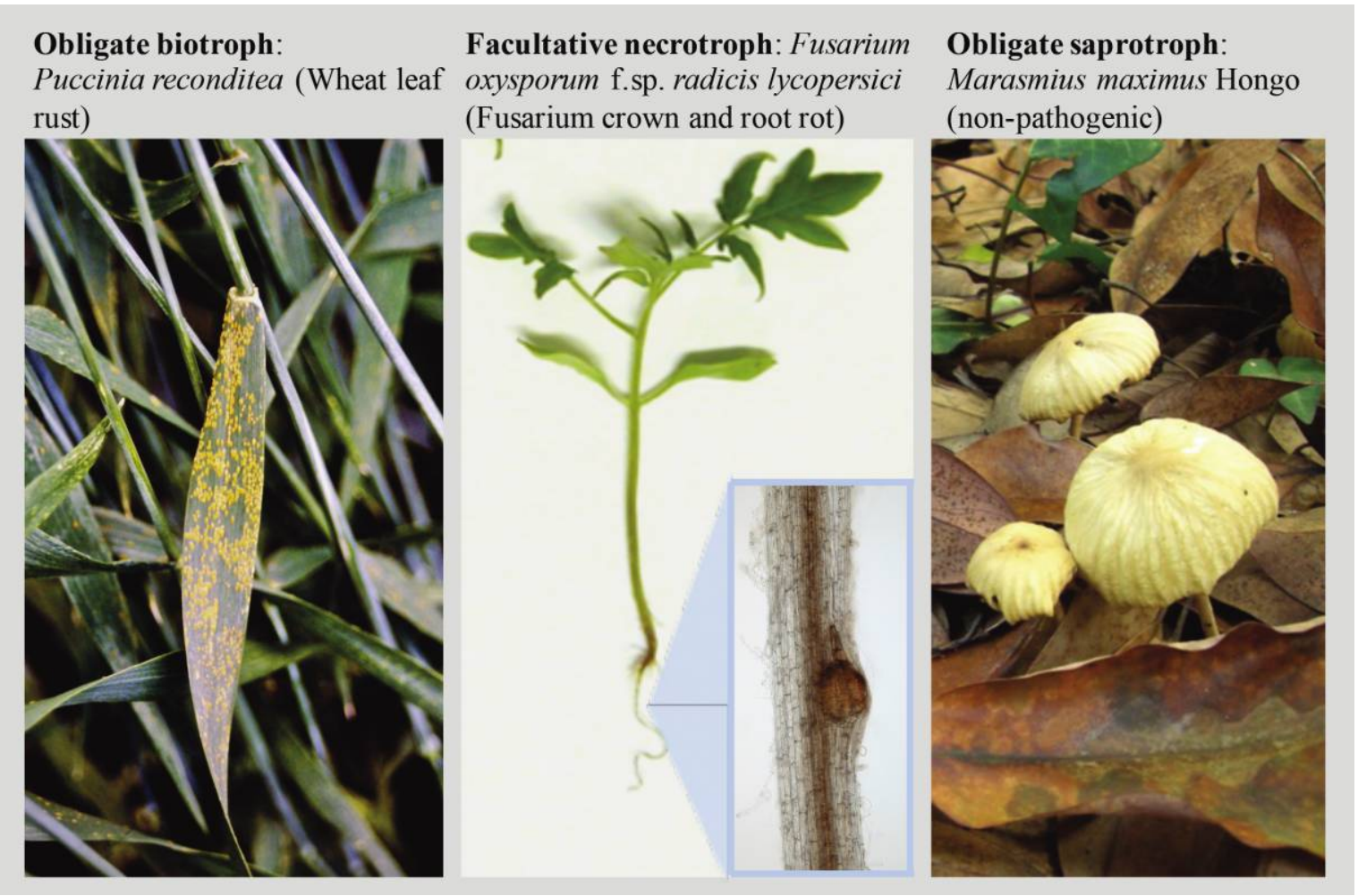

Examples of three types of fungi (bio-/necro-/saprotroph) based on their trophic strategies. Puccinia reconditea parasitizes living wheat leaves. Fusarium oxysporum invades the host vascular system (center; vascular browning occurs in the tomato root) and then causes wilting of the entire seedling. Marasmius maximus absorbs nutrients from litter in a forest environment. Photo credits: Yasuo Ohto (left); Sayaki U. Suzuki (center, right). 